\section{Uber die Entwicklung der Chirurgie wäh- rend des 19. Jahrhunderts und ihre Beziehung zum Unterricht.}

Akademische Rede zur Feier des Geburtsfestes des höchstseligen Grobherzogs Karl Friedrich am 21. November 1903 bei dem Vortrag des Jahresberichts und der Verkündung der akademischen Preise.

Gehalten von Dr. Vincenz Czerny, Wirkl. Geh. Rat und

o. ö. Professor der Chirurgie, d. Z. Prorektor der GroBh. Bad.

Universität Heidelberg.

(Fortsetzung aus No. 1.)

Sehr wesentlich wurden diese Studien durch das Tierexperiment unterstützt und gefördert. Schon Immanuel Kant ${ }^{1}$ ) hat die Bedingungen, unter welchen das Experiment Erfolg haben kann, scharf formuliert: "Die Vernunft mun mit ihren Prinzipien, nach denen allein übereinkommende Erscheinungen für Gesetze gelten können. in der einen Hand. mit dem Experiment, das sie nach jenen ausdachte, in der anderen an die Natur gehen, zwar um von ihr belehrt zu werden, aber nicht in Gestalt des Schülers, der sich alles vorsagen läßt, was der Lehrer will, sondern eines bestallten Richters, der die Zeugen nötigt, auf die Fragen zu antworten, die er ihnen vorlegt." Im Experiment können wil uns willkürlich die Bedingungen schaffen und dadurch die Natur zwingen. auf unsere Fragen Antwort zu geben.

Schon Harvey entdeckte den Blutkleislauf, Albrecht von Haller die Muskeliritabilität durch das Experiment. John Hunter machte Versuche über Übertragung von vollständig abgetrennten Körperteilen, die wieder anheilten. Charles Bell entdeckte durch das Tierexperiment den Unterschied der sensiblen und motorischen Nervenwurzeln, die großen französischen Experimentalphysiologen, vor allen Magendie und Claude Bernald, an die sich unsere deutsche Physiologenschule von Johannes Müllel, Karl Ludwig bis auf Kühne anschlossen, haben durch das Tielexperiment den stolzen Bau unserel Kenntnisse über die physiologische Tätigkeit der Organe errichtet. Noch in jüngster Zeit hat Pawloff in Petersburg in dem Institute, welches der Hel'zog von Oldenburg gegründet hat. die Lehre von der Verdaung durch seine Versuche an Hunden aufgeklärt. Tie Versuche von Heine in Würzburg ïber die Neubildung von Knochengewebe durch das Periost gaben Bernhard von Langenbeck den Anstof zu seinen subperiostalen Resektionen, welche im Schleswig-Holsteinischen Kriege zuerst in grölierem Malistabe zur Ausfïhrung gekommen sind und bei richtiger Nachbehandlung ausgezeichnete Resultate herbeigeführt haben.

Gustav Simon hat an Tieren festgestellt, daß der Ausfall einel Niere durch die Funktion der anderen kompensiert werden könne und hat es gewagt, das Resultat dieses Versuches mit glänzendem Erfolge auf den kranken Menschen zu übertragen und dadurch den Anstoß zu dem großen Gebiete der Nierenchirurgie zut geben.

Billroth und seine Schule förderten durch Tierexperimente unsere durch die Franzosen Jobert und Lembert angebahnten Kenntnisse über die beste Nahtmethode bei Verletzungen des Darmes, studierten die teilweise oder gänzliche Ausschneidung des Magens und eröffneten dadurch neue Gebiete der Unterleibschirurgie.

So könnte ich hunderte von Tatsachen anführen, aus denen unwiderleglich hervorgeht, daf das von manchen Seiten viel verlästerte Tierexperiment nicht nur unsere Kenntnisse sehr wesentlich gefördert hat, sondern auch hundertfachen Nutzen für die Behandlung der Krankheiten und zur Linderung der dem ganzen Menschengeschlechte beschiedenen Qualen geschaffen hat. So lange die Menschen Millionen von Tierleben opfern, um ihren materiellen Hunger zu stillen, wird man auch das mit möglichster Schonung des Schmerzgefühls ausgeführte Tierexperiment zur Stillung des Wissensdurstes gestatten müssen. Der Drang nach der Erforschung der Wahrheit ist nicht weniger quälend, als der materielle Hunger und Durst. Es hätten sonst nicht hunderte von Märtyrern für ihre Überzeugung Lebensglück und Gesundheit hingeopfert und sich dem Märtyrertode geweiht.

Auch die genauere Kenntnis der schmerzstillenden Mittel, welche unendlich viel zur Verminderung und Abschwächung der

1) Kant, Forrede zur zweiten Ausgabe der Kritik der reinen Vernunft, geschrieben im April 1787. Berlin, Heimann, 1870, S. 26. alle chirurgischen Eingriffe begleitenden Schrecknisse beigetragen haben, verdanken wir im wesentlichen dem Tierexperimente, wenn auch die Anfänge derselben auf zufällige Beobachtungen beim Menschen zurückzuführen sein dürften. Die Empfindungslosigkeit des Menschen im Alkoholrausch ist sicher eine Jahrhunderte alte Erfahrung. Humphry Davy benutzte die damals neue Kenntnis der Gase zu therapeutischen Zwecken und lief Sauerstoff, Stickoxydul, welchem er den Namen Lachgas gab. und sogar auch Schwefeläther zur Beseitigung von asthmatischen Beschwerden einatmen. Aber erst der Chemiker und Arzt Jackson und der Zahnarzt Morton in Boston empfahlen 1846 systematisch die Anwendung der Athernarkose zum Zwecke der schmerzlosen Ausführung von Operationen. Die Amerikaner beschenkten die alte Welt mit der künstlichen Erzeugung der Schmerzlosigkeit und dürfen mit Stolz ihren später so unglücklichen Landsleuten die Devise aufs Grab setzen: Jovi dolorem eripuerunt.

Es wiirde mich $\mathrm{zu}$ weit führen, hier des Genaueren auseinander zu setzen, wie der Äther von dem Schotten Simpson durch das Chloroform ersetzt worden ist, wie die alten Methoden der lokalen Anästhesie durch Kältewirkung wieder durch neue Mittel, wie die $\ddot{A}$ therzerstäubung, zur Anwendung gekommen sind, wie man nach Erkenntnis der Gefahr, welche die allgemeine Anästhesie als eine Art Vergiftung in sich birgt, dieselbe zu ersetzen suchte durch lokale Anästhetica, wie die Auffindung des Cocaïns und seiner synthetischen Ersatzmittel immer mehr dazu führt, den schmerzstillenden cheinischen Kern von den giftigen Stoffen zu isolieren, und wie die merkwürdigen Produkte, welche man aus der Nebenniere gewonnen hat, imstande sind, diese lokal anästhesierende Wirkung in wunderbarer Weise zu steigern. Tatsache ist. dail alle diese noch imıner im Flusse befindlichen Untersuchungen unserer Kenntnisse über die Funktion der Nerven, über den eigentümlichen Reiz derselben, den wir als Schmerz empfinden, auferordentlich vertieft haben, dali aber auch die Beseitigung der Schmerzempfindung es uns erınöglicht hat, operative Eingriffe auszuführen, vor denen noch wenige Jahr zehnte vorher die kühnsten Chirurgen zurückgeschreckt wären.

Dahin gehört vor allen anderen die enorme Entwicklung der Chirurgie der Unterleibsorgane. Noch in seiner Operativen Chirurgie ${ }^{1}$ ) hat Dieffenbach, der kühnste und geschickteste Chirurg in der Mitte des vorigen Jahrhunderts, die Ausführıng der Ovariotomie als ein tollkühnes Unternehmen bezeichnet, das weder dem Klanken, noch auch dem Operateur Segen bringen könne. Lennoch hatte schon der amerikanische Arzt Mac Dowell seit dem Jahre 1809 mit Absicht und Erfolg mehrere Ovariotomien ausgefuhrt. Seine Berichte wurden aber nicht beachtet. Die spärlichen Versuche, ihm nachzuahmen, hatten elst in den Händen von Spencer Wells in London und Köberlé in Strassburg durchschlagenden Erfolg. Erst die Einführung der antiseptischen Wundbehandlung durch Lord Joseph Lister hat den mit Eröffnung des Bauchfells verbundenen Operationen ihre Gefahr genommen und den Erfolg aller operativen Eingriffe so sehr gesichert, daß die Zahl derselben gegen früher nicht nur verhundertfacht worden ist, sondern die Prognose des Eingriffs als solchen sich mit mathematischer Genauigkeit nach den bisherigen Erfahrungen auf dem Gebiete vorausbestimmen läßt. Zufällige Wundkrankheiten, Eitervergiftungen, septisches Wundfieber, Rotlauf, Starrkrampf, welche früher oft zu den einfachsten Eingriffen hinzukamen, lassen sich durch die Einführung der antiseptischen und aseptischen Wundbehandlung von nicht infizierten Wunden mit fast vollkommener Sicherheit abhalten. ${ }^{2}$ )

Die Mortalität der Ovariotomie, der Amputationen, der komplizierten Knochenbrüche, welche früher $50-60 \%$ betrug, ist durch die Listersche Entdeckung und ihre Ausbildung auf 5-6\% gesunken. Viele Operationen, welche man früher wegen der großen Lebensgefahr kaum auszuführen wagte, gehören jetzt zu den täglichen Aufgaben des Chirurgen. So die Exstirpation des Kropfes, die uralte Trepanation, welche fast vergessen war, wurde erweitert zul Gehirnchirurgie und diente nicht allein zum Aufsuchen von Abszessen, zur Entfernung von Knochensplittern, sondern auch zur Beseitigung von Geschwiilsten in der Gehirnoberfläche. Die dabei gemachten Erfahrungen erweiterten unsere Kenntnisse über die Lokalisation der Gehirnfunktion. Auch der

) Die operative Chirurgie. Bd. II. Leipzig 1848. S. 802.

2) Ad. Kehrer, Über die Vorgänge bei der Wundbehandlung. Akademische Rede. Heidelberg 1898. 
Räckgratskanal wurde eröffnet und verborgene Geschwülste, welche aus den Symptomen richtig diagnostiziert werden konnten, beseitigt und in manchen Fällen dem Rückenmark seine Leistungsfähigkeit wieder zurückgegeben. Die operative Behandlung der Ergüsse in die Brusthöhle, ja selbst in den Herzbeutel wurde wieder aufgenommen, methodisch ausgebildet und führte viel häufiger zu einem günstigen Erfolge, als vorher. Durch die Radikaloperation der Unterleibsbrüche werden Tausende von jungen Menschen von dem lästigen und unsicheren Bruchbande befreit und Hunderte wieder dienstfähig fürs Militär und leistungsfähig für schwere Arbeit. Als ganz neues Gebiet wurde die operative Beseitigung der Gallensteine, wenn sie allen Bemühungen der inneren Medizin zum Trotze nicht abgehen wollen und ihrem Träger grole Beschwerden und Gefahren verursachen, durchgeführt. Die älteren Versuche, Leberabszesse und Echinokokken operativ anzugreifen, wurden mit glücklichem Erfolge wieder aufgenommen und selbst Geschwülste aus der Leber entfernt, wobei die Experimente von Ponfick die merkwürdige Regenerationsfähigkeit der Lebersubstanz nachgewiesen haben. Zu den schon oben erwähnten Operationen am Magen- und Darmkanal, die durch die chemische und mechanische Untersuchung des Inhalts und der Lage mit der Magenpumpe außerordentlich an Sicherheit gewonnen haben, gesellte sich die operative Behandlung der Blinddarmentzündung, als deren Ausgangspunkt der Wurmfortsatz erkannt wurde, dessen Beseitigung die großen Gefahren der Erkrankung in der Regel aufhebt. Die Operation ist leider beinahe Mode geworden, zum Teil weil durch die operativen Eingriffe die früher sehr ungewisse Diagnose viel sicherer gemacht worden ist, zum Teil aber auch, weil die Erkrankung teils durch die Lebensweise, teils vielleicht durch die wiederholten Influenzaepidemien häufiger geworden ist. Auch die Operationen an der Blase und an der Niere haben bedeutend an Sicherheit gewonnen und ihre Erkennung durch Erfindung des Blasenspiegels grobe Fortschritte gemacht.

Wenn der Unterschied in der Gesamtheit der Mortalität nach Operationen von jetzt gegen früher nicht so auffallend sich gebessert hat, wie ich es oben für die Amputation und die Ovariotomie hervorgehoben habe, so liegt es daran, daf immer neue und schwierigere Operationsgebiete erobert worden sind und dah die erzielten Erfolge zu Eingriffen ermutigten, bei denen die Aussichten nur geringe sein konnten. Selbst der Kranken bemächtigt sich der Gedanke, daßs, wenn alle Hilfsmittel nichts nützen, vielleicht durch eine Operation noch geholfen werden könne, und gar manchmal lälit sich der Operateur dadurch zu einem Eingriffe bestimmen, den er bei genauer Kenntnis der Sachlage lieber unterlassen hätte. Das vorgerückte Alter gilt im allgemeinen nicht mehr als eine Gegenanzeige operativer Eingriffe, aber dennoch können wichtige Organe des Kreislaufes und der Lunge so abgebraucht sein, dafi Komplikationen von dieser Seite einen Strich durch die beste Berechnung machen.

Lister ging bei der Entdeckung seiner Behandlungsmethode von den Untersuchungen Pasteurs aus, welcher nachwies, daß die Zersetzung von Flüssigkeiten ausbleibt, wenn man den Hinzutritt von organischen Keimen verhindert. In dieser Beziehung hatte Pasteur schon Vorgänger, da Schwann, v. Helmholtz, Schröder und v. Dusch durch ähnliche Experimente denselben Beweis, wenn auch vielleicht nicht so augenfällig, geliefert hatten. Lister wurde durch den auffallenden Unterschied im Heilungsverlaufe von einfachen und komplizierten Knochenbrüchen dazu geführt, daß die so viel größere Gefahr bei den offenen Knochenbrüchen durch das Hinzutreten der Luft und von Zersetzungserregern bedingt sein müsse. Er suchte deshalb die Luft und die Wunde zu desinfizieren und benutzte dazu als bestes Antiseptikum die Karbolsäure. Mit divinatorischem Scharfblicke erkannte er aber auch die Wichtigkeit, die Hände und Instrumente vor der Berührung mit anderen infektiösen Stoffen in acht zu nehmen und die mechanischen Insulte der Gewebe bei den Operationen auf das möglichst geringste $\mathrm{Maß}$ einzuschränken. Wie er sich ausdrückte, solle man die Wunde allein lassen, wenn sie gut heilen solle. Auch Lister hatte in dieser Beziehung schon einen Vorgänger, da schon vorher der Geburtshelfer Semmelweiss in Wien die Überzeugung ausgesprochen hatte, daß das in den Gebärkliniken so gefährliche verheerende Wochenbettfieber durch Übertragung von fauligen Stoffen, namentlich vermittelst der Hände der Ärzte und Hebammen zustande komme, und dall extreme Sauberkeit und möglichst wenig Berüh- rung der Gebärenden diese Gefahren erheblich einschränken oder beseitigen könne.

Schon Lister hat sich bemüht, seine Methode nach verschiedenen Richtungen zu modifizieren, aber die wesentlichste Vereinfachung und experimentelle Begründung hat dieselbe in Deutschland gefunden. Es wurde durch v. Bruns und v. Mikulicz festgestellt, dab die Gefahr der Luftinfektion verhältnismäßig gering sei, daß man den Karbolspray Listers entbehren kann, dab die antiseptischen Mittel nicht nur Gifte für die Bakterien, sondern auch für die Gewebe des menschlichen Körpers sind und dab man auch ohne dieselben, mit strengster Reinlichkeit, Desinfektion der Instrumente und Verbandstoffe, durch Hitze und strömenden Dampf imstande ist, die besten Heilungsresultate $\mathrm{zu}$ erzielen. Infolgedessen ist die aseptische an die Stelle der antiseptischen Methode getreten (v. Bergmann). Da es sich herausgestellt hat, daß auch bei der größten Vorsicht und gründlichsten Desinfektion eine vollkommene Keimfreiheit der Wunde nicht zu erzielen ist, trotzdem aber der Heilungsverlauf sich regelmäßig aseptisch gestaltet, hat man es gelernt, den im lebenden Körper vorhandenen Schutzkräften gegen die Infektion größ3eren Wert beizulegen und die mechanische Insultierung der Gewebe bei den Operationen auf das möglichst geringste Maßs einzuschränken.

Bei diesen Studien über die Ursachen der Wundinfektion, welche namentlich durch Theodor Billroth und Otto Weber mit Zuhilfenahme der damals von Bärensprung u. a. ausgebildeten Thermometrie eingeleitet worden sind, hat man das zahllose Heer der Bakterien namentlich mittelst der durch Rober Koch verfeinerten Kulturmethoden genauer kennen gelernt. Man hat gefunden, daß sie zwar durch ihre ungemein rasche Vermehrungsfähigkeit die Gefäß3e und Gewebe schädigen und durch ihre Stoffwechselprodukte den Organismus vergiften, dali sie aber andererseits bei ungünstigen Lebensbedingungen leicht zu Grunde gehen oder doch ihre Gefährlichkeit einbüßen. Das genaue Studium derselben auf künstlichen Nährböden im Tierexperimente hat diesen kleinen Unholden, wie z. B. den Pestbazillen, einen großen Teil ihres Schreckens genommen, wenn auch mancher Experimentator seinen weniger vorsichtigen Umgang mit denselben durch Selbstinfektion mit dem Tode büßien mußte. ${ }^{1}$ ) Das genaue Studium der mit diesen Mikroben infizierten Tiere hat ergeben, daß bei vielen eine gewisse Angewöhnung eintreten kann und daß\} die Tiere eine Immunität gegen weitere Ansteckung mit diesen Mikroben gewinnen. ${ }^{2}$ ) Diese Tatsache, welche sich an die alte klinische Erfahrung, dafS das Überstehen einer Infektionskrankheit, wie Blattern, Scharlach, Masern, vor einer zweiten Erkrankung meistens schützt, führte dazu, aus den immunisierten Tieren Schutzstoffe zu gewinnen, welche sowohl die Tiere selbst gegen solche Seuchen sichern, als auch den Menschen wie durch einen Impfstoff gegen diese Krankheiten immunisieren oder durch hochpotenzierte Schutzstoffe von der schon ausgebrochenen Krankheit wieder befreien können.

Den glänzendsten Erfolg auf diesem Gebiete hat Behring durch die Entdeckung des Diphtherieserums erzielt. Die friiher so gefürchtete Diphtherie hat bei seiner rechtzeitigen Anwendung den grö̈ten Teil ihres Schreckens verloren und durch die fortgesetzte Bekämpfung auch ihre frühere Gefahr zum Teil eingebüit. Hier in Heidelberg ist durch die Einführung des Diphtherieserums die Behandlung der diphtheritischen Kinder und die jetzt seltener gewordene Tracheotomie fast vollständig von der chirurgischen Klinik auf die Kinderklinik übergegangen. Es ist sehr erfreulich, zu sehen, dali die modernen Fortschritte der Therapie auch wieder manche Gebiete für die innere Behandlung zurückerobern, während immer neue Gebiete innerer Krankheiten der mechanischen, das ist der chirurgischen Behandlung, zugeführt werden.

Die stets zunehmende Verwertung physikalischer und chemis cher Untersuchungsmethoden für die Diagnose der Krank-

3) Zu den zahllosen Aerzten, die Wundintektionen bei Operationen und Sektionen zum Opfer gefallen sind, gresellen sich die Laboratoriums infektionen beim Studium der Bakterien. Nur aus jïngster Zeit nenne ich in Wien: Heider durch Typhusbazillen, Hoffmann v. Wellen hof (Rotz), Müller (Pest); in Berlin: Knorr (Rotz), Sachs (Pest), Myers (gelbes Fieber), Oergel (Hamburg), Pfeifer (Königsberg) Pfuhl an Cholera Marx (Frankfurt a. M.) an Typhus. - 2) J. Arnold Ueber den Kampf des menschlichen Körpers mit den Bakterien. Aka demische Rede, Heideiberg 1888. 
heiten ist eine wesentliche Ursache, daß sich von dem Hauptstamm der Chirurgie rerschiedene wichtige Seitenzweige selbständig entwickelt und im Laufe des Jahrhunderts abgetrennt haben. Bis zur Erfindung des Augenspiegels durch Hermann v. Helmholtz beschränkte sich die Augenheilkunde wesentlich auf die Behandlung der äußeren Teile des Sehorgans bis zur Linse. Dieses beschränkte Gebiet konnte der Chirurg neben seinen verhältnismäßig einfachen sonstigen Aufgaben noch bewälltigen. Nachdem aber $v$. Helmholtz wie mit einem Schlage das Innere des Auges dem staunenden Blick bis in den verborgensten Winkel bloßgelegt hatte, nachdem er in seiner physiologischen Optik die mathematischen Probleme der Dioptrie auf die Refraktionsanonalien des Auges anzuwenden gelehrt hatte, da stellten sich im Zusammenhange mit der durch Heinrich Müller, Max Schulze, Briicke, Leber u. a. geförderten mikroskopischen Anatomie des Auges eine solclie Fülle von neuen Problemen ein, daß sie bloß durch geniale, unermüdliche Spezialisten gelöst werden konnten. Im rechten Monente trat die Lichtgestalt Alblecht v. Gräfes auf, und wie die Veredelung eines alten Baumes durch ein neues Pfropfreis wurde die gesamte Heilkunde durch die Entwicklung der Augenheilkunde fruchtbringend beeinflutit.

Auch die Erfindung des Kehlkopfspiegels, der von Czermak und Türk in Wien für die Praxis nutzbal gemacht worden ist, ermöglichte die lokale Behandlung del Kehlkopfleiden unter Leitung des Gesichtes. Die ersten operativen Erfolge, welche Viktor' v. Bruns in Tübingen auf dieseln Gebiete erzielte, veranlałten die Abzweigung einer neuen spezialität, die sehr bald die Erkrankungen der Luftröhre, del Nasen- und Rachenhöhle sich hinzugesellte.

Auch die Ohrenheilkunde, welche lange etwas stiefmütteriich im Nebenamte von der Chirurgie verwaltet wurde, erhielt durch die Erfindung des Olirenspiegels durch Toynbee und durch die systematische Bearbeitung ihrer physikalischen Grundlagen durch Helmholtz mächtige Impulse und hat in den Händen gewiegter Spezialisten den alten Skeptizismus durch glänzende Erfolge überwunden. Lurch kühne Opelationen an Warzenfortsatz, Behandlung der gefahrdrohenden Eiterungen in den benachbarten Blutleitel'n und Gehirnpartien haben die Ohrenärzte und Chirurgen sich gegenseitig befruchtet.

In Deutschland ist die operative Gynäkologie, für welche Gustav Simon in Heidelberg bedeutsame Fortschritte angebahnt hat, wesentlich an die Geburtshelfer übergegangen und hat sich in deren Händen inächtig entwickelt. In anderen Ländern führt sie entweder ein selbständiges Dasein oder wird mehr von Chirurgen ausgeübt.

Die mechanische Belıandlung von Verkrümmungen der Extremitäten durch Maschinen, wie sie namentlich Zander in vorzüglicher Weise erdacht hat, die Wiederaufnahme der Massage, welche von den Römern geübt und im Oriente niemals ganz vergessen war, die damit vielfach verknüpfte Hydrotherapie, die Heißluft- und Lichtbäder. die Anwendung von Elektrizität, die schwierige Verbandtechnik sind die Veranlassung, da\} auch die Orthopädie sich neben der Chirurgie selbständig entwickelt und neue Gebiete, namentlich aus der Nervenpathologie, eroberte. Die Anwendung der Röntgenphotographie, die uns so wichtige Dienste bei der Behandlung ron Knochenbrüchen und Verrenkungen, bei der Entfernung von Fremdkörpern leistet, hat sich zu einer schwierigen uud auch kostspieligen Technik ausgebildet, welche die Zeit und Intelligenz eines geschickten Mitarbeiters reichlich in Anspruch nimmt.

So sehen wir, daß die Chirurgie mit der Ausdehnung ihres Machtbereiches auch wieder Einbuße erleidet dadurch, daß neue Spezialitäten sich von ihr abzweigen, die alleldings in ihrer selbständigen Entwicklung wieder mächtig zur Fortbildung der Heilkunde beitragen. Aber wie jedes Ding in der Welt zwei Seiten hat, so stehen auch hiel dem Lichte manche Schatten gegenüber. So viel auch Deutschland zu der Entwicklung der Chirurgie beigetragen hat, so ist es doch kein bloßer Zufall, dal die zwei gröften Erfindungen, welche den mächtigen Aufschwung der Chirurgie ermöglicht haben: die Einführung der allgemeinen Narkose und die antiseptische Wundbehandlung nicht bei uns, sondern in Amerika und England gemacht worden sind.

(Schluß folgt.) 